\title{
Low vision management in a case of stargardt's disease
}

\begin{abstract}
Background: By the WHO definition "a person with low vision is a one who has impairment of visual functioning even after treatment, surgery or standard refractive correction and has a visual acuity of less than $6 / 18$ to light perception or a visual field of $<10^{\circ}$ from the point of fixation, in the better eye but who is potentially able to use vision for the planning and execution of a task." Stargardt's disease is one of the commonest inherited retinal disorders, with a prevalence of 1 in 10,000. It is inherited as an autosomal recessive trait. ${ }^{1,2}$ Most cases present with central visual loss and there is typically macular atrophy and with yellowwhite flecks at the posterior pole, which are at the level of the retinal pigment epithelium (RPE). Autofluorescence (AF) imaging and fluorescein angiography can be helpful in the confirming the diagnosis. ${ }^{3,4}$ The age of onset is usually in the early teens, but there is wide variation, with a later age of onset being associated with a better visual prognosis. ${ }^{5,6}$
\end{abstract}

Case: A 26 year old female presented to the low vision clinic of B.P. Koirala Lions Center for Ophthalmic Studies after final diagnosis of B/L Stargardt's disease and her presenting visual acuity of $0.5 \log$ MAR in RE and $0.56 \log$ MAR in her LE. She had no previous history of any ocular pathology, trauma in the past. She had no history of any systemic illness known to her and no similar history of vision loss in other family members. Her visual acuity was decreasing since 8 years which was apparently well 8 years back. The anterior segment was within normal limits and on dilated fundus examination on mydriasis there was typical honeycomb appearance of the macular region. On cycloplegic retinoscopy there was final subjective prescription of -0.50 DC X 180 axis but no improvement in her visual acuity in her both eyes. Her near visual acuity was N12 at 33cm of working distance. With the spectacle clip on telescope $(1.5 \mathrm{X})$ her visual acuity improved to $0.20 \log$ MAR. $54 \%$ yellow filter as spectacle was also prescribed and approach magnification was also taught. Finally the patient was managed with $1.5 \mathrm{X}$ spectacle clip on telescope for distance, bar magnifier (1.5X) for near and 54\% yellow filter for management of photophobia.

Conclusion: Stargardt's disease can be managed properly with improvement in visual acuity for both near and distance with significant improvement in visual acuity for both near and distance with significant improvement in other visual functions with low vision devices.

Keywords: low vision management, stargardt's disease, telescope, bar magnifier, filter
Volume 2 Issue I - 2015

\section{Pabita Dhungel, ND Joshi}

Department of Ophthalmology, Tribhuvan University, Teaching Hospital, Nepal

\author{
Correspondence: Pabita Dhungel, Department of \\ Ophthalmology, BP Koirala Lions Centre for Ophthalmic \\ Studies, Institute of Medicine, Tribhuvan University Teaching \\ Hospital Maharajgunj, Kathmandu, Nepal, \\ Email pabitadhungel@yahoo.com
}

Received: December 30, 2014 | Published: January 21, 2015
Abbreviations: ERG, electro-retinogram; RPE, retinal pigment epithelium; AF, auto fluorescence

\section{Introduction}

Vision rehabilitation as defined by American Optometric Association is the process of treatment and education that helps individuals who are visually disabled attain maximum function, a sense of well-being, a personally satisfying level of independence, and optimum quality of life. Low vision rehabilitation cannot restore the lost sight but helps the patient with his/her remaining vision to maintain an independent life style. Stargardt's disease affects individuals of any age, gender and race. ${ }^{1,5,7}$ Limit of visual acuity, fundus appearance and severity of the disease varies widely among patients. ${ }^{1,78}$ Typically, fundus flecks and atrophic patches at the macula and the mid-peripheral retina are observed with a variable degree of macular dysfunction alone and additional loss of cone or cone rod function may or may not be present. ${ }^{1,7-10}$ Fundus autofluorescence observed by fundus photograph has emerged as the most useful non-invasive tool for the evaluation of the patients with Stargardt's disease. ${ }^{9,11,12}$ By means of autofluorescence imaging both active and resorbed flecks can be clearly visualized and appear as foci of very high or low autofluorescence signal, respectively. Areas of retinal pigment epithelial atrophy can also be identified as areas of very low autofluorescence signal at the macula and mid-peripheral retina, the latter commonly associated with reduced peripheral cone and rod function and, thus, they will have poorer visual prognosis. ${ }^{9,12,13}$ Fundus autoflourescence imaging can also be used to monitor the progression of the disease by demonstrating the development or disappearance of areas on increased autofluorescence, the expansion of pre-existing areas of reduced autofluorescence, or the occurrence of new ones. ${ }^{14,15}$ Visual acuity can be variable and have a range from 20/20 to 20/400 with associated central visual field scotoma. ${ }^{5,16}$ The difficulties in performing daily activities such as reading and driving have been evaluated by previous studies. ${ }^{17,18}$ Low vision management provides the patients with optimal use of the residual visual acuity. For the first time, Stargardt disease was described as the most common forms of juvenile macular degeneration in 1909 . It contributed to $13.9 \%$ of the causes of low vision in age under 16 years. ${ }^{19}$ Stargardt's disease may occur in one of every 20,000 children over the age of 6 year and is usually diagnosed before the age of 20 years. Both the genders are equally affected by this condition. Affected person has difficulty in recognizing faces, reading, writing and other close works, such that the affected can see the objects with peripheral or side vision. 
Peripheral vision is useful for moving about with premises. The disease is diagnosed by teenage. ${ }^{7,20}$ The progression of disease in the retina as well as in area of central vision tends to be extensive over time. The sharpness of vision may decrease and blind spots may be denser when the patient is exposed to bright light and the retina is bleached by the light. The problem of glare and photophobia are temporary and can be prevented or lessened by the use of sun filters and hats. The optimistic side of this disease is that it never causes total vision loss and peripheral vision remains intact. The low vision examination will evaluate the person's functional use of remaining vision. A comprehensive, interdisciplinary approach of optometrist, ophthalmologist and rehabilitation workers can help the majority of low vision people learn to use their remaining visual capacity to its fullest and be independent as possible to live a better life. This approach includes persons, who have best corrected visual acuity of $8 / 18$ or less, and/or visual field of 20 degrees or less.

\section{Case report}

A 26 year old female presented to our low vision clinic referred from retina clinic of the same department of B.P Koirala Lions Center for Ophthalmic Studies with the provisional diagnosis of B/L Stargardt's disease. She had no previous history of wearing glasses. She had diminution in vision of her both eyes slow and progressive in nature since 8 years. Her eyes were apparently well 8 years back. She sustained no previous history of any systemic illness and no prior ocular trauma and disease. She had no similar history of visual loss in her other family members. Her presenting visual acuity was 0.50 $\log$ MAR in RE and $0.56 \log$ MAR in her LE. On examination by binocular slit lamp examination, the anterior segments were within normal limits but on fundus examination under mydriatics revealed typical features of Stargardt's disease.

\section{Low vision examination}

She was accompanied by her brother during the examination. She was married and a mother of two children and her children had no visual problems. She was bachelor level student with no previous history of glasses. Her family tree suggests 5 siblings, she was second child to her parents and rest of the children were apparently well. She had no previous history of low vision examination. She had problem with near vision task regarding difficulties with newspaper and college books. She had no difficulties in writing and was able to write in a straight line. On distance vision task, she had difficulties in recognizing faces, watching television, bus and tempo numbers. On specific lighting need examination, she was comfortable with reduced light and fluorescent lamp. She had mobility problems in outdoors and unfamiliar places. She suggested no any history of bumping into objects. She had no ocular preference between her two eyes. She had glare problem more pronounced in sunlight and car light. She had history of delayed light adaptation in daylight condition. She had no history of colour perception problem. She was comfortable in her daily living skills like home management, self-help skills, money identification, food identification and any other.

Her chief visual problems were:
i. Blurring of distance vision
ii. Difficulty in identifying small prints
iii. Difficulty in bright day light

Her distance visual acuity was $0.50 \log \mathrm{MAR}$ in her right eye and $0.56 \operatorname{logMAR}$ in her left eye. Her near vision was N12 unaided at
$33 \mathrm{~cm}$ of reading distance. On objective retinoscopy her refractive error was +1.00/-0.50X 180 in RE and +1.00/-0.50 X 180 in LE with no significant improvement in visual acuity in her both eyes.

\section{Binocularity assessment}

Extraocular motility was clinically full ranged and cover test revealed no shift. The contrast sensitivity tested with Pelli-Robson Chart was 1.6 logunits in her both eyes which signifies poor contrast sensitivity. The visual field as tested by confrontation test was within normal limits.

\section{On trial of near and distance vision devices}

Patient was prescribed bar magnifier (1.5X) for near visual task of reading. She was comfortable using it reading and near visual acuity was improved to N8 for near which was optimum vision for near task. Patient's response was good and her visual acuity improved significantly upto $0.20 \operatorname{logMAR}$ with $1.5 \mathrm{X}$ spectacle clip on telescope. Her visual skills were good on searching things around her with the use of telescope. She was also taught about the proper handling techniques of spotting, tracing, tracking and scanning methods with the use of telescope.

\section{On trial of absorptive lenses}

Several absorptive lenses were tried subjectively on patient. Polarized lenses were also tried. Acceptance was best with the 54\% yellow filter as tested in daylight illumination. The visual objectives were accomplished with the use of absorptive lenses that the patient was more comfortable with it.

\section{Rehabilitation plan}

i. Spectacle correction with clip on telescope (1.5X)

ii. Bar magnifier (1.5X) for near vision.

iii. $54 \%$ yellow filter on spectacle.

iv. Table lamp with fluorescent direct light for near evaluation.

v. Patient was asked to follow up every three months for low vision evaluation.

\section{Discussion}

Low vision is equivalent to visual impairment, and it has historically been referred to by other terms, such as subnormal vision or partial sight. It refers to a condition of diminished visual performance that cannot be rectified by surgical methods, medical means, or with the use of conventional spectacles or contact lenses. Reduced visual function may also be the result of an abnormal contrast sensitivity function. It is difficult to identify a person having low vision strictly on the basis of visual acuity, because the term low vision specifically implies a reduction in function. Performance on a high-contrast acuity test may or may not accurately reflect an individual's ability to function in the environment. Generally speaking, reduced visual function may also be a result of decreased contrast sensitivity, decreased colour vision, abnormal ocular motility, sensitivity to glare, or decreased binocularity.

In Stargardt's disease, the cone cells, situated at the macula, deteriorate in function and eventually die. The rod cells generally remain intact. Symptoms usually start at a young age- usually under 20 years. People with Stargardt's disease start to experience difficulty with reading and fine handwork as well as distinguishing colours. 
These symptoms progress with age. In some, this deterioration is rapid, while in others it is much slower. There is no way of detecting how fast this deterioration will occur in any individual. By the age of 50 , about half will have a visual acuity of $6 / 60$ or worse. People with Stargardt's disease do not usually have a problem with bumping into objects when moving around. They may however experience difficulties in adjusting to light. The diagnosis is usually made as a result of deterioration in visual acuity and typical appearances around the macula on ophthalmoscopy. Electrical tests, such as the electroretinogram (ERG), electro-oculogram and dark adaptation testing can measure the progress of the disease, but are not usually necessary for diagnosis. Low vision services improve the quality of life and mental state. ${ }^{21,22}$ Clinical trials providing evidence of effectiveness of specific interventions for individuals with functional low vision are lacking. ${ }^{23}$ Further research is recommended to compare different types of low vision devices according to the underlying cause and need of the individual.

\section{Conclusion}

Stargardt's patients respond well to magnification. The central visual functioning part, the macula is more affected eccentric viewing techniques helps in patients. As a part of non-optical devices the filters help in patient in the management of glare and photophobia. The low vision aid should be changed as the disease progresses and the patient should be on regular follow up. Limitation of this study was multiple case reports should have to be taken instead of single case study. A long term prospective study that carefully monitors the magnitude and quality of low vision is needed to add to our knowledge of this type of low vision case.

\section{Acknowledgments}

None.

\section{Conflicts of interest}

Author declares that there is no conflict of interest.

\section{References}

1. Stargardt K. Uber familiare progressive degeneration in der maculagegend des auges. Albrecht von Graefes Archiv für Ophthalmologie. 1909;71(3):534-550.

2. Michaelides M, Hunt DM, Moore AT. The genetics of inherited macular dystrophies. J Med Genet. 2003;40(9):641-650.

3. Lois N, Halfyard AS, Bird AC, et al. Fundus autoflourescence in Stargardt macular dystrophy-fundus flavimaculatus. Am J Ophthalmol. 2004;138(1):55-63.

4. von Ruckmann A, Fitzke FW, Bird AC. In vivo fundus autofluoresence in macular dystrophies. Arch Ophthalmol. 1997;115(5):609-615.

5. Rotenstreich Y, Fishman GA, Anderson RJ. Visual acuity loss and clinical observations in a large series of patients with Stargardt disease. Ophthalmology. 2003;110(6):1151-1158.

6. Walia S, Fishman GA. Natural history of phenotypic changes in Stargardt macular dystrophy. Ophthalmic Genet. 2009;30(2):63-68.
7. Klien BA, krill AE. Fundus Flavimaculatus. Clinical, functional, and histopathologic observations. Am J Ophthalmol. 1967;64(1):3-23.

8. Lois N, Holder GE, Bunce C, et al. Phenotypic subtypes of Stargardt macular dystrophy-fundus flavimaculatus. Arch Ophthalmol. 2001;119(3):359-369.

9. Fishman GA. Fundus flavimaculatus. A clinical classification. Arch Ophthalmol. 1976;94(12):2061-2067.

10. Oh KT, Weleber RG, Oh DM, et al. Clinical phenotype as a prognostic factor in Stargardt disease. Retina. 2004;24(2):254-262.

11. Lois N, Holder GE, Fitzke FW, et al. Intrafamilial variation of phenotype in Stargardt macular dystrophy-Fundus flavimaculatus. Invest Ophthalmol Vis Sci. 1999140(11):2668-2675.

12. Lois N, Halfyard AS, Bird AC, et al. Fundus autofluoresence in Stargardt macular dystrophy- fundus flavimaculatus. Am J Ophthalmol. 2004;138(1):55-63

13. McBain VA, Lois N. Fundus autofluorescence in Stargardt disease. In: Lois N \& Forrester JV, editors. Fundus Autofluorescence. $1^{\text {st }}$ editin, Philadelphia, PA: Lippincott, USA, 2009. p. 206-216.

14. Chen B, Tosha C, Gorin MB, et al. Analysis of autofluorescent retinal images and measurement of atrophic lesion growth in Stargardt disease. Exp Eye Res. 2010;91(2):143-152.

15. Smith RT, Gomes NL, Barile G, et al. Lipofuscin \& autofluorescence metrics in progressive Stargardt disease. Invest Ophthalmol Vis Sci. 2009;50(8):3907-3914.

16. Sunness JS, Applegate CA, Haselwood D, et al. Fixation patterns and reading rates in eyes with central scotomas from advanced atrophic agerelated macular degeneration and Stargardt disease. Ophthalmology. 1996;103(9):1458-1466.

17. Szlyk JP, Fishman GA, Grover S, et al. Difficulty in performing everyday activities in patients with juvenile macular dystrophies: comparison with patients with retinitis pigmentosa. $\mathrm{Br} J$ Ophthalmol. 1998;82(12):1372-1376.

18. Szlyk JP, Fishman GA, Severing K, et al. Evaluation of driving performance in patients with juvenile macular dystrophies. Arch Ophthalmol. 1993;111(2):207-212.

19. Shah M, Khan MD. Causes of low vision amongst the low-vision patients attending the Low-Vision Clinic at Khyber Institute of Ophthalmic Medical Sciences (KIOMS), Hayatabad Medical Complex Peshawar, Pakistan. Vis Impair Res. 2004;6:89-97.

20. Moloney JB, Mooney DJ, O'Connor MA. Retinal function in Stargardt's disease and fundus flavimaculatus. Am J Ophthalmol. 1983;96(1):57-65.

21. Vijayakumar V, John RK, Datta D, et al. Quality of life after communitybased rehabilitation for blind persons in a rural population of south India. Indian J Ophthalmol. 2004;52(4):331-335.

22. Horowitz A, Reinhardt JP, Boerner k. The effect of rehabilitation on depression among visually disabled older adults. Aging Ment Health. 2005;9(6):563-570.

23. Reeves BC, Harper RA, Russell WB. Enhanced low vision rehabilitation for people with age related macular degeneration: a randomized controlled trial. Br J Ophthalmol. 2004;88(11):1443-1449. 